\title{
A Unified Mixed Logit Framework for Modeling Revealed and Stated Preferences: Formulation and Application to Congestion Pricing Analysis in the San Francisco Bay Area
}

\author{
Chandra R. Bhat and Saul Castelar \\ Dept of Civil Engineering, ECJ 6.8, University of Texas at Austin, Austin, Texas, 78712 \\ Phone: (512) 471-4535, Fax: (512) 475-8744 \\ Email: bhat@mail.utexas.edu, scastelar@mail.utexas.edu
}

\begin{abstract}
This paper formulates and applies a unified mixed-logit framework for joint analysis of revealed and stated preference data that accommodates a flexible competition pattern across alternatives, scale difference in the revealed and stated choice contexts, heterogeneity across individuals in the intrinsic preferences for alternatives, heterogeneity across individuals in the responsiveness to level-of-service factors, state dependence of the stated choices on the revealed choice, and heterogeneity across individuals in the state dependence effect. The estimation of the mixed logit formulation is achieved using simulation techniques that employ quasi-random Monte Carlo draws. The formulation is applied to examine the travel behavior responses of San Francisco Bay Bridge users to changes in travel conditions. The data for the study are drawn from surveys conducted as part of the 1996 San Francisco Bay Area Travel Study. The results of the mixed logit formulation are compared with those of more restrictive structures on the basis of parameter estimates, implied trade-offs among level-of-service attributes, heterogeneity and state dependence effects, data fit, and substantive implications of congestion pricing policy simulations.
\end{abstract}

Keywords: Revealed preference, stated preference, mixed logit, quasi-Monte Carlo simulation, state dependence, unobserved heterogeneity, congestion pricing. 


\section{INTRODUCTION}

Stated preference data (self-stated preferences for market products or services) have been widely used in the marketing and travel demand fields, separately or in conjunction with revealed preference data (observed choices of product purchase or service use), to analyze consumers' evaluation of multi-attributed products and services. Stated preference (SP) and revealed preference (RP) data each have their own advantages and limitations with respect to estimation of behavioral parameters of interest (Ben Akiva et al., 1992; Hensher et al., 1999). This realization has led to the now long history of using both kinds of data simultaneously to analyze consumer behavior (e.g., Gunn et al. 1992, Ben-Akiva and Morikawa 1990; Koppelman et al., 1993,Swait and Louviere, 1993; Hensher et al., 1999).

Four important issues need to be recognized in joint RP-SP estimation: (a) interalternative error structure, (b) scale difference between the RP and SP data generating processes, (c) unobserved heterogeneity effects, and (d) state dependence effects and heterogeneity in the state dependence. Each of these is discussed in turn in the subsequent four sections. Section 1.5 presents the need to consider all of the issues simultaneously within a unified RP-SP modeling framework.

\subsection{Inter-Alternative Error Structure}

The revealed choice and stated choice observations represent the decisions of individuals to choose one alternative from among a set of alternatives. Within a random utility maximization framework, the mathematical expression for the choice probability of an alternative at any choice occasion depends on the covariance structure assumed for the random error terms across alternatives. A common assumption is that the error terms are identically and independently 
(IID) distributed across alternatives with a type I extreme value distribution. This leads to the simple and elegant closed-form multinomial logit (MNL) model. However, the IID error structure assumption also leaves the MNL model saddled with the "independence of irrelevant alternatives" (IIA) property at each choice situation (Luce and Suppes, 1965; see also Ben-Akiva and Lerman, 1985 for a detailed discussion of this property).

The literature on joint RP-SP methods has, with few exceptions, assumed a MNL structure for the RP and SP choice processes. However, with recent methodological advances, RP-SP methods can be quite easily extended to accommodate flexible competitive patterns by relaxing the IID error structure across alternatives, using closed-form model formulations such as the Generalized Extreme Value (GEV) family of models (see Koppelman and Sethi, 2000) or more general open-form model formulations such as the mixed-multinomial logit family of models (see Bhat, 2000).

Recent studies in the joint RP-SP literature that accommodate non-IID inter-alternative error structures include Hensher et al. (1999) and Brownstone et al. (2000). The former study accommodates heteroscedasticity across alternatives within the framework of Generalized Extreme Value (GEV) models, while the latter accommodates both heteroscedasticity and correlation across alternatives within the framework of a mixed multinomial logit model.

\subsection{Scale Difference}

The RP and SP choices are made under different circumstances; RP choices are revealed choices in the real world, while SP choices are stated choices made in an experimental and hypothetical setting. In both the real world and experimental settings, the analyst does not have information on all the factors that influence an individual's choice. These unobserved (to the analyst) factors 
are usually subsumed within the random error term of the utility function. Thus, the unobserved factors in an RP setting can include individual decision-maker factors, unmeasured alternative attributes, and measurement error in variables. The unobserved factors in an SP setting can include unobserved individual factors, omission of relevant variables affecting the choice context under examination, and characteristics of the experimental design. Since the RP and SP choice settings are quite different, there is no reason to believe that the variance of the unobserved factors in the RP setting will be identical to that of the variance of unobserved factors in the SP setting (see Ben-Akiva and Morikawa, 1990). There is also no a priori theoretical basis to suggest whether the RP error term or the SP error term will have the larger variance; this may be closely tied to the empirical context under examination.

The scale difference between the RP and SP choice contexts has been recognized and accommodated in almost all previous joint RP-SP analyses.

\subsection{Unobserved Heterogeneity Effects}

Unobserved heterogeneity effects refer to unobserved (to the analyst) differences across decision-makers in the intrinsic preference for a choice alternative (preference heterogeneity) and/or in the sensitivity to characteristics of the choice alternatives (response heterogeneity). Stated preference methods usually involve experimental settings in which each of a sample of individuals is exposed to different stimuli corresponding to different combinations of values for the set of explanatory variables under study. It is at least possible (if not very likely) that the responses from the same individual to the different stimuli will be affected by common unobserved attributes of the individual. Ignoring these attributes is tantamount to assuming away the presence of individual-specific unobserved effects, which can result in inconsistent SP model 
parameter estimates and even more severe inconsistent choice probability estimates (see Chamberlain, 1980; the reader is also referred to Hsiao, 1986 and Diggle et al., 1994 for a detailed discussion of heterogeneity bias in discrete-choice models).

Of course, unobserved heterogeneity effects are not confined to the SP choice responses. The same unobserved individual-specific attributes influencing the SP choices made by an individual will also affect the RP choice of the individual. These unobserved attributes generate a correlation in utility for an alternative across all choice occasions (RP and SP choices) of the individual. The unobserved heterogeneity effects also lead (indirectly) to non-IID error structures across alternatives at each choice occasion, so that the IIA property does not hold at any choice occasion.

Most RP-SP studies in the literature disregard unobserved heterogeneity. However, Morikawa (1994) accommodates unobserved preference heterogeneity in his analysis by considering an error-components structure for the RP and SP error terms. But Morikawa's study does not accommodate unobserved response heterogeneity (i.e., differences in sensitivity to characteristics of the choice alternatives). Hensher and Greene (2000) have recently accommodated unobserved response heterogeneity, along with inter-alternative correlation, in a study on vehicle type choice decisions.

\subsection{State Dependence and Heterogeneity in State Dependence}

State dependence, in the context of joint RP-SP estimation, refers to the influence of the actual (revealed) choice on the stated choices of the individual (the term "state dependence" is used more broadly here than its typical use in the econometrics field, where the term is reserved specifically for the effect of actual past choices on actual current choices). State dependence 
could manifest itself as a positive or negative effect of the choice of an alternative on the utility associated with that alternative in the stated responses. A positive effect may be the result of habit persistence, inertia to explore another alternative, or learning combined with risk aversion (i.e., the individual is familiar with the attributes of the chosen alternative and feels "safe" choosing it in subsequent choice situations). It could also be the result of justification for the RP choice. A negative effect could be the result of variety seeking or the result of latent frustration with the inconvenience associated with the currently used alternative (for example, a captive transit user may prefer a proposed rail alternative over the current bus alternative). Further, in most choice situations, it is possible that the effect of state dependence is positive for some individuals and negative for others (see Ailawadi et al., 1999). Besides, even within the group of individuals for which the effect is positive (or negative), the extent of the inertial (or varietyseeking) impact on stated choices may vary. Thus, joint RP-SP estimations should not only recognize state dependence, but also accommodate heterogeneity in the state dependence effect.

Most RP-SP studies in transportation disregard state dependence. No study in the literature, to the authors' knowledge, recognizes and accommodates unobserved heterogeneity in the state dependence effect of the RP choice on SP choices (Brownstone et al., 1996 accommodate observed heterogeneity in the state dependence effect by interacting the RP choice dummy variable with sociodemographic attributes of the individual and SP choice attributes).

\subsection{A Unified RP-SP Modeling Framework}

The earlier four sections have discussed the need to consider a flexible inter-alternative error structure, RP-SP scale difference, unobserved heterogeneity, and state dependence. In this 
section, we supplement the discussion in the previous sections by highlighting the need to consider all these four issues jointly within a unified RP-SP modeling framework.

The fundamental reason for considering all the four modeling issues simultaneously is that there is likely to be interactions among them. Thus, accommodating restrictive interalternative error structures rather than flexible error structures can lead to misleading behavioral conclusions about taste effects and scaling effects in joint RP-SP models (see Hensher et al., 1999; Louviere et al., 1999). Similarly, adopting restrictive inter-alternative structures can overstate unobserved heterogeneity in a model, and ignoring unobserved heterogeneity can overstate inter-alternative error correlations. It is also imperative that unobserved heterogeneity be incorporated in a model with state dependence (see Heckman, 1981; Keane, 1997). In the context of joint RP-SP estimation, if unobserved heterogeneity exists and the analyst ignores it, the unobserved heterogeneity can manifest itself in the form of spurious state dependence; that is, the effect of the RP choice on SP choices may be artificially overstated. ${ }^{1}$ Similarly, if the RP choice affects SP choices and the analyst ignores this state dependence, the state dependence will manifest itself in the form of unobserved heterogeneity and overstate the level of unobserved heterogeneity. In addition, ignoring state dependence or unobserved heterogeneity can, and generally will, lead to a bias in the effect of other coefficients in the model (Heckman, 1981; Hsiao, 1986).

In this paper, we propose a unified RP-SP framework that adopts a mixed multinomial logit formulation to accommodate all of the four modeling considerations discussed above. The

\footnotetext{
${ }^{1}$ Econometrically speaking, the RP choice variable is correlated with the error term in the SP choice equation in the presence of unobserved heterogeneity. This issue is similar to the initial conditions problem in the panel data literature (Chamberlain, 1980; Degeratu, 1999).
} 
paper is structured as follows. The next section presents the model formulation. Section 3 discusses model estimation. Section 4 describes the data sources and the sample used in empirical analysis. Section 5 presents the results of the empirical analysis. Section 6 applies the estimated models to examine the potential effects of congestion-pricing policies. The final section concludes the paper by highlighting important findings from the analysis.

\section{MODEL FORMULATION}

The utility $U_{q i t}$ that an individual $q$ associates with an alternative $i$ on choice occasion $t$ may be written in the following form ( $t$ may represent the RP choice occasion or an SP choice occasion):

$\left.U_{q i t}=\alpha_{q}^{\prime} x_{q i t}+\theta_{q}\left[\left(1-\delta_{q t, R P}\right) \times\left(\sum_{s=1}^{T_{q}} \delta_{q s, R P} Y_{q i s}\right)\right)\right]+\varepsilon_{q i t}$,

where $x_{\text {qit }}$ is a vector of observed variables (including variables characterizing the RP and SP alternative specific constants), $\alpha_{q}$ is a corresponding coefficient vector which may vary over individuals but does not vary across alternatives or time, $\delta_{q t, R P}$ is a dummy variable taking the value 1 if the $t^{\text {th }}$ choice occasion of individual $q$ corresponds to her/his revealed preference choice and 0 otherwise, $Y_{q i t}$ is another binary variable that takes a value of 1 if the individual $q$ chooses alternative $i$ at the $t^{\text {th }}$ choice occasion and 0 otherwise, $T_{q}$ is the total number of observed choice occasions for individual $q, \theta_{q}$ is the individual-specific state dependence effect that maps the effect of the RP choice of an alternative into the utility evaluation of that alternative in the SP choice occasions, and $\varepsilon_{q i t}$ is an unobserved random term that captures the idiosyncratic effect of omitted variables during each choice occasion. $\varepsilon_{\text {qit }}$ is assumed to be independent of $\alpha_{q}$ and $x_{q i t}$. The reader will note that the second term in equation (1), 
representing the state dependence effect, reduces to zero in the utility evaluation for the RP choice occasion.

The error term $\varepsilon_{q i t}$ may be partitioned into two components, $\zeta_{q i t}$ and $\mu^{\prime} z_{q i t}$. The first component, $\zeta_{q i t}$, is assumed to be independently and identically Gumbel distributed across alternatives and individuals for each choice occasion, and also independently (but not identically) distributed across choice occasions. Its scale parameter is specified as: $\lambda_{q t}=\left[\left(1-\delta_{q t, R P}\right) \times \lambda\right]+\delta_{q t, R P}$. Such a specification accommodates the scale difference between the RP and the SP choice occasions. Specifically the RP scale is normalized to one for identification, and the SP scale, $\lambda$, is estimated. The second component in the error term, $\mu^{\prime} z_{\text {qit }}$, induces heteroscedasticity and correlation across unobserved utility components of the alternatives at any choice occasion $t . z_{\text {qit }}$ is a vector of observed data, some of whose elements might also appear in the vector $x_{q i t} \cdot \mu$ is a random multivariate normal vector with zero mean. For example, $z_{\text {qit }}$ may be specified to be a row vector of dimension $M$ with each row representing a group $m(m=1,2, \ldots, M)$ of alternatives sharing common unobserved components. The row(s) corresponding to the group(s) of which $i$ is a member take(s) a value of one and other rows take a value of zero. The vector $\mu$ (of dimension $M$ ) may be specified to have independent elements, each element having a variance component $\sigma_{m}^{2}$. The result of this specification is a covariance of $\sigma_{m}^{2}$ among alternatives in group $m$ and heteroscedasticity across the groups of alternatives. This structure is less restrictive than the nested logit structure in that an alternative can belong to more than one group (or nest). Also, by structure, the variance of the alternatives is different. More general structures for $\mu^{\prime} z_{\text {qit }}$ in equation (1) are presented by Ben-Akiva and Bolduc (1996), and Brownstone and Train (1999). 
Several special structures can be obtained by imposing appropriate restrictions on equation (1). For instance, assuming that $\alpha_{q}=\alpha$ and $\theta_{q}=0$ for each individual $q$, and assuming that $\mu=0$, implies a multinomial logit structure for the RP-SP choices with a scaling effect, but without state dependence and unobserved heterogeneity effects. If the assumption that $\mu=0$ is relaxed in the above structure, the IID specification across alternatives is relaxed. In addition, if $\alpha_{q}$ is not constrained to be the same, but allowed to vary across individuals, unobserved heterogeneity is added to the specification. Next, if the assumption that $\theta_{q}=0$ is relaxed and it is assumed that $\theta_{q}=\theta$, state dependence is included in the specification. Finally, if the restriction that $\theta_{q}$ is the same across individuals is also removed, we get the most general model that accommodates flexible inter-alternative error structure, allows the scale to be different between RP and SP responses, incorporates unobserved heterogeneity in the parameters, and recognizes state dependence and heterogeneity in state dependence.

$$
\text { Defining } \beta_{q}=\left(\alpha_{q}^{\prime}, \theta_{q}\right)^{\prime}, w_{q i t}=\left[x_{q i t}^{\prime},\left(1-\delta_{q t, R P}\right) \times\left(\sum_{s=1}^{T_{q}} \delta_{q s, R P} Y_{q i s}\right)\right]^{\prime} \text {, and using the error }
$$

components decomposition for $\varepsilon_{\text {qit }}$ discussed above, equation (1) can be re-written as:

$$
U_{q i t}=\beta_{q}^{\prime} w_{q i t}+\mu^{\prime} z_{q i t}+\zeta_{q i t}
$$

The coefficient $\beta_{q}$ in equation (2) is individual-specific and, in general, varies across individuals. Let the distribution of unobserved heterogeneity across individuals be multivariate normal, so that $\beta_{q}$ is a realization of a random multivariate normally distributed variable $\widetilde{\beta}$. Let $\omega$ be a vector of true parameters characterizing the mean and variance-covariance matrix of $\widetilde{\beta}$. 
Further, let $\sigma$ be a parameter vector characterizing the variance-covariance matrix of the multivariate normal distribution of $\mu$.

Conditional on $\widetilde{\beta}$ and $\mu$, the probability that individual $q$ will choose alternative $i$ at the $t^{\text {th }}$ choice occasion can be written in the usual multinomial logit form (see McFadden, 1978):

$$
P_{q i t} \mid(\widetilde{\beta}, \mu)=\frac{e^{\lambda_{q t}\left(\tilde{\beta}^{\prime} w_{q i t}+\mu^{\prime} z_{q i t}\right)}}{\sum_{J=1}^{I} e^{\lambda_{q t}\left(\tilde{\beta}^{\prime} w_{q i t}+\mu^{\prime} z_{j i t}\right)}}
$$

The unconditional probability can be subsequently obtained as:

$$
P_{q i t}=\int_{\tilde{\beta}=-\infty}^{+\infty} \int_{\mu=-\infty}^{+\infty} \frac{e^{\lambda_{q t}\left(\tilde{\beta}^{\prime} w_{q i t}+\mu^{\prime} z_{q i t}\right)}}{\sum_{J=1}^{I} e^{\lambda_{q t}\left(\tilde{\beta}^{\prime} w_{q j t}+\mu^{\prime} z_{q i t}\right)}} d F(\mu \mid \sigma) d F(\widetilde{\beta} \mid \omega),
$$

where $F$ is the multivariate cumulative normal distribution. The reader will note that the dimensionality in the integration above is dependent on the number of elements in the $\mu$ and $\beta_{q}$ vectors.

\section{MODEL ESTIMATION}

The parameters to be estimated in the model of equation (4) are the $\sigma$ and $\omega$ vectors. To develop the likelihood function for parameter estimation, we need the probability of each sample individual's sequence of observed RP and SP choices. Conditional on $\widetilde{\beta}$, the likelihood function for individual $q$ 's observed sequence of choices is:

$$
L_{q}(\widetilde{\beta}, \sigma)=\prod_{t=1}^{T_{q}}\left[\int_{\mu=-\infty}^{+\infty}\left\{\prod_{i=1}^{I} P_{q i t}(\widetilde{\beta}, \mu)^{Y_{q i t}}\right\} f(\mu \mid \sigma) d \mu\right]
$$

The unconditional likelihood function of the choice sequence is: 


$$
\begin{aligned}
L_{q}(\omega, \sigma) & =\int_{\tilde{\beta}=-\infty}^{+\infty} L_{q}(\tilde{\beta}, \sigma) f(\tilde{\beta} \mid \omega) d \tilde{\beta} \\
& =\int_{\tilde{\beta}=-\infty}^{+\infty}\left\{\prod_{t=1}^{T_{q}}\left[\int_{\mu=-\infty}^{+\infty}\left\{\prod_{i=1}^{I} P_{q i t}(\tilde{\beta}, \mu)^{Y_{q i t}}\right\} f(\mu \mid \sigma) d \mu\right]\right\} f(\tilde{\beta} \mid \omega) d \tilde{\beta} .
\end{aligned}
$$

The log-likelihood function is $\mathscr{L}(\omega, \sigma)=\Sigma_{q} \ln L_{q}(\omega, \sigma)$.

The likelihood function in equation (6) is quite different from those in previous applications of the mixed logit model, such as Bhat (1998), Hensher (2000), and Brownstone and Train (1999). In particular, there are two levels of integration rather than one. This arises because, from an estimation standpoint, the error components formulation that generates interalternative correlation operates at the choice level, while the random coefficients formulation that accommodates taste variation across individuals operates at the individual level. Thus, in the context of estimating such a bi-level structure, error components and random coefficients are not mathematically equivalent.

We apply quasi-Monte Carlo simulation techniques to approximate the integrals in the likelihood function and maximize the logarithm of the resulting simulated likelihood function across all individuals with respect to $\omega$ and $\sigma$. The procedure to simulate each individual's likelihood function, $L_{q}(\omega, \sigma)$, is as follows: (a) For a given value of the parameter vector $\omega$, draw a particular realization of $\widetilde{\beta}$ from its distribution, (b) For a given value of the $\sigma$ vector, draw several sets of realizations of $\mu$ from its distribution, each set corresponding to a choice occasion of the individual, (c) compute the probability of the chosen alternative for each choice occasion (i.e., the likelihood function of that choice occasion) at that choice occasion's set of $\mu$ realizations, and for the current $\widetilde{\beta}$ realization, (d) Average the likelihood functions across the various realizations of $\mu$ for each choice occasion, (e) Compute the individual likelihood 
function as the product of the averaged likelihood functions across all choice occasions of the individual, (f) Repeat steps a through e several times with fresh realizations of $\widetilde{\beta}$ and new sets of draws of $\mu$, and (g) Compute the average across all individual likelihood function evaluations. Mathematically, the individual likelihood function is approximated as:

$$
S L_{q}(\omega, \sigma)=\frac{1}{D} \sum_{d=1}^{D}\left[\prod_{t=1}^{T_{q}}\left\{\frac{1}{G} \sum_{g_{d}=1}^{g_{d}=G}\left(\prod_{i=1}^{I} P_{q i t}\left(\tilde{\beta}^{d}\left|\omega, \mu^{g_{d}}\right| \sigma\right)^{Y_{q i t}}\right)\right\}\right],
$$

where $S L_{q}(\omega, \sigma)$ is the simulated likelihood function for the $q^{\text {th }}$ individual's sequence of choices given the parameter vector $\omega$ and $\sigma, \widetilde{\beta}^{d} \mid \omega$ is the $d^{\text {th }} \operatorname{draw}(d=1,2, \ldots, D)$ from $f(\widetilde{\beta} \mid \omega), \mu^{g_{d}} \mid \sigma$ is the $g_{d}{ }^{\text {th }}$ draw $\left(g_{d}=1,2, \ldots, G\right)$ from $f(\mu \mid \sigma)$ at the $d^{\text {th }}$ draw of $\widetilde{\beta}$, and other variables are as defined earlier. $S L_{q}(\omega, \sigma)$ is an unbiased estimator of the actual likelihood function $L_{q}(\omega, \sigma)$. Its variance decreases as $D$ and $G$ increase. It also has the appealing properties of being smooth (i.e., twice differentiable) and being strictly positive for any realization of the draws.

The simulated log-likelihood function is constructed as:

$S \mathscr{L}(\omega, \sigma)=\sum_{q} \ln \left[S L_{q}(\omega, \sigma)\right]$

The parameter vector $\omega$ and $\sigma$ are estimated as the values that maximize the above simulated function. Under rather weak regularity conditions, the maximum (log) simulated likelihood (MSL) estimator is consistent, asymptotically efficient, and asymptotically normal (see Hajivassiliou and Ruud, 1994; Lee, 1992; McFadden and Train, 1998).

In the current paper, we use the Halton sequence to draw realizations for $\beta$ and $\mu$ from their population normal distributions. Details of the Halton sequence and the procedure to generate this sequence are available in Bhat, (1999). Bhat (1999) has demonstrated that the Halton simulation method out-performs the traditional pseudo-Monte Carlo (PMC) methods for 
mixed logit model estimation. Subsequent studies by Train (1999) and Hensher (1999) have confirmed this result.

The estimations and computations in the paper were carried out using the GAUSS programming language on a personal computer. Gradients of the log simulated likelihood function with respect to the parameters were coded.

\section{DATA SOURCES AND SAMPLE FORMATION}

The data for the study are drawn from revealed preference (RP) and stated preference (SP) surveys conduced as part of the 1996 San Francisco Bay Area Travel Study (BATS). The surveys were designed and administered by NuStats Research and Consulting for the Metropolitan Transportation Commission (NuStats Research and Consulting, 1996).

The objective of the RP survey was to provide data for the continued development and refinement of travel demand models for the San Francisco Bay area. This RP survey included a 48-hour travel diary of all members of sampled households, and collected detailed individual and household socio-demographic information. The objective of the SP survey was to obtain additional data to model the travel behavior responses of Bay Area Bridge users to changes in travel conditions, including changes in bridge tolls, parking costs, travel times, transit fares, and transit service frequency. The main emphasis, however, of the SP survey was to provide data for congestion pricing models in the Bay Bridge corridors. Individuals who were 18 years or older, participated in the RP survey, and who reported crossing one of the Bay Bridges at least once a week during the peak hours of 6-9 a.m., were eligible for participation in the SP survey. The final recruitment of individuals for the SP survey from this "eligible pool" was based on the availability of a reported trip using one of the Bay Bridge corridors during the 48-hour diary 
period of the RP survey. This reported trip in the RP survey served as the reference trip for development of the SP scenarios. The reference trip, along with the "current travel conditions" corresponding to the available travel modes for the reference trip, were then presented to each SP survey respondent. Finally, hypothetical scenarios of new travel conditions for the reference trip were generated and the corresponding choices of respondents were recorded. The reader is referred to NuStats Research and Consulting (1996) for complete details of survey sampling and administration procedures.

The experimental design for the SP survey generated 32 scenarios of "new travel conditions", representing changes from the reference trip scenario. The 32 scenarios were further grouped into four sets of eight questions each, and each survey respondent was randomly assigned one of the four sets. The SP survey collected information from 150 respondents. Of these respondents, 14 respondents had to be removed from the sample because they did not complete the SP survey or had missing information on travel conditions. The final sample comprises 136 respondents. Of these 136 respondents, 130 contributed 9 choice occasions (8 SP choice occasions + 1 RP choice occasion). However, some of the SP choice occasions of the remaining 6 respondents had to be discarded because the respondents chose a transit travel mode that is not available to them for their reference trip. Among these six respondents, two respondents contributed seven choice occasions, while one each of the remaining four contributed 3,4,5, and 8 choice occasions. Thus, the final sample comprises a total of 1,204 choice occasions (136 RP choice occasions and 1,068 SP choice occasions).

The universal choice set includes six alternatives: 1) Drive alone or with one other person during the peak period (DAP), 2) Drive alone or with one other person during the off-peak period (DAO), 3) Carpool (3 or more people) during the peak period (CPP), 4) carpool during 
the off-peak, period (CPO), 5) Alameda County Transit (ACT), and 6) Bay Area Rapid Transit (BART). The peak period is defined as 6 a.m. to 9 a.m. and 3 p.m. to 6 p.m. The first four of the above alternatives are available at each choice occasion of each individual. However, the ACT transit mode is not available for 73 respondents (= 561 choice occasions) and the BART transit mode is not available for 13 respondents (= 107 choice occasions). Both these transit modes are unavailable for 10 respondents (= 86 choice occasions).

The following information was provided to respondents for the reference trip for the transit modes (BART and ACT): availability of the modes, headway of transit service, total travel time, and transit fare. The information provided on the non-transit alternatives for the reference trip included total travel time and bridge tolls. However, current total travel costs for the non-transit alternatives were not presented for the reference trip. This was developed by the authors using several supplementary data sources. The level-of-service values for the SP scenarios were constructed based on the values for the reference trip and the incremental changes specified in each SP scenario.

The choice share of alternatives in the RP sample, in the SP sample, and in the joint sample is provided in Table 1 (for ease in presentation, we will use the label "drive alone" for the "drive alone or with one other person" mode and the label "carpool" for the "carpool with 3 or more individuals" mode). The RP sample indicates a higher mode share captured by the transit modes compared to the $10 \%$ transit market share for work trips in the Bay area. This is because the destination for about $91 \%$ of the trips in the current RP sample is San Francisco. A comparison of the RP and SP mode shares indicates a clear shift away from the drive alone-peak (DAP) mode toward the drive alone-off peak (DAO) and carpool-peak (CPP) modes. This is, in part, because the SP choice scenarios involve a steep increase over the RP scenario in peak- 
period bridge toll and parking costs (the increase in cost ranged from zero to $\$ 8$, with a mean increase of \$3.25). The substantial shift may also be attributed to individuals overstating their likelihood to switch in their SP responses.

\section{EMPIRICAL ANALYSIS}

A number of different model structures were estimated in the analysis. In this paper, we present the results of seven model structures: four "cross-sectional" models that do not accommodate unobserved heterogeneity and state dependence, and three "panel-data" models that accommodate unobserved heterogeneity and/or state dependence. In the models with state dependence, we included only the modal dimension effect of the RP choice on SP choices. Alternative specifications that included the temporal dimension of state dependence were estimated, but did not provide significant temporal dependence parameters. This result is to be expected, since a very small fraction of the drive alone and carpool users in the RP sample travel in the off-peak hours (see Table 1). Finally, mode-specific state dependence parameters were also tested, but we could not reject the hypothesis of a generic state dependence effect. This is perhaps reflecting the dominance of the drive alone mode share in the RP sample.

\subsection{Cross-Sectional Models}

Table 2 provides the results of the cross-sectional models. The first model is the "RP-Sample MNL" model, the second is the "SP-Sample MNL" model, the third is a "joint RP/SP-MNL" model, and the fourth is a "joint RP/SP error-components" model.

The coefficients on the alternative-specific constants show considerable differences between the RP and SP samples due to differences in the alternative shares in the two samples. 
In particular, the coefficients on the drive alone off-peak (DAO) and carpool peak (CPP) alternatives are much more positive in the SP sample relative to their counterparts in the RP sample. This suggests that individuals are overstating their tendency to shift to the DAO and CPP alternatives from the drive alone peak (DAP) alternative in the SP experiments.

The signs of the coefficients on other variables in the different models are quite reasonable. The effects of the socio-demographic variables indicate that individuals in households with a high number of vehicles relative to number of workers are likely to choose the drive alone modes (DAP or DAO). Also, individuals who are male and employed tend to choose the drive alone mode during the peak period than other travel alternatives. Finally, within the category of socio-demographic effects, the results suggest that high income earners are likely to choose the drive alone mode during the peak period and the ACT mode (when it is available to them) compared to other travel alternatives. Among the level-of-service variables, the results show the expected negative effects of total travel times and costs (in all the models, the headway of transit service was introduced as an additional variable, but provided a counter-intuitive positive sign and was statistically insignificant; hence it is dropped from the model specifications).

A comparison of the "RP-Sample MNL" model with the other cross-sectional models shows differences in the level-of-service variable effects. The "RP-Sample MNL" model has a statistically insignificant cost coefficient. This reflects the limited variation in cost within the RP sample as well as multi-collinearity between time and cost. The other cross-sectional models provide a statistically significant cost coefficient.

The "joint RP/SP-MNL" model and the "joint RP-SP error components" model show that there is no significant scale difference in the RP and SP choice processes (see the last but one 
row in Table 2). For the error components model, we considered several different interalternative error structures. However, we found that a simple specification allowing a common error component for the drive alone modes (DAP and DAO) and another common error component for the shared-ride modes (CPP and CPO) adequately captured the inter-alternative error structure. Further, we could not reject the hypothesis of equal variances of the drive alone and shared-ride error components (see last row of Table 2). The resulting inter-alternative error structure implies a higher degree of sensitivity between the drive alone modes than between a drive alone mode and a non-drive alone mode. Similarly, the structure also implies a higher degree of sensitivity between the carpool modes than between a carpool mode and a non-carpool mode. More fundamentally, these results suggest that individuals are more likely to change departure times than change travel modes in response to changes in travel service characteristics. The error structure discussed above also generates heteroscedasticity between the transit and non-transit alternatives; specifically, the variance of the non-transit alternatives (DAP, DAP, CPP, and CPO) is larger than the variance of the transit alternatives. Such a heteroscedastic pattern implies a higher degree of sensitivity between the transit alternatives than between a transit and a non-transit alternatives. In addition, the heteroscedasticity pattern also implies that the self-sensitivity of transit alternatives to transit level-of-service changes is higher than the self-sensitivity of non-transit alternatives to non-transit level of service changes (see Bhat, 1995 for a detailed discussion of the competitive patterns implied by heteroscedasticity).

Table 3 provides the implied money values of travel time and fit statistics from the various models. The implied money value of time is very high in the "RP-Sample MNL" model. This is because the "RP-Sample MNL" model is unable to capture the sensitivity to cost due to limited variation in cost within the RP sample as well as multi-collinearity between time and 
cost. The implied money value is more reasonable in the other three models, though still on the high side for urban travel.

In addition to the four models in Table 3, we also estimated an unrestricted "joint RP/SPMNL" model with a completely different set of coefficients in the RP and SP choice processes, except for the cost coefficient, which was constrained to be the same to identify the RP-SP scale difference. This model had a convergent log-likelihood value of -1614.25. A comparison of this value with the log-likelihood value at convergence for the "joint RP/SP-MNL" model in Table 2 suggests that, after accommodating for constant differences, there are no taste differences in the $\mathrm{RP}$ and SP choice processes. The likelihood ratio value for the test is 8.8 , which is smaller than the chi-squared value corresponding to the 6 additional parameters in the unrestricted model at a 0.1 level of significance.

In summary, the results of the cross-sectional models indicate that the joint RP/SP approach is better able to represent trade-offs in level-of-service attributes and also provide efficiency benefits in estimation by recognizing the presence of a common latent preference structure underlying the RP and SP responses. The joint RP/SP approach is also able to recognize and adjust for the overstatement in use of the drive alone off-peak and carpool peak alternatives in the SP responses. Among the two cross-sectional joint RP/SP models, the errorcomponents formulation provides a statistically better data fit. Thus, it is the preferred crosssectional model structure and is retained as the base structure for the development of the panel data models. 


\subsection{Panel Data Models}

Several "panel data" models were estimated in the analysis. In the current paper, we present only three of these to keep the discussion focused and also due to space considerations. The first model accommodates unobserved heterogeneity effects (both heterogeneity in intrinsic preferences and in response to level-of-service variables), but not state dependence. The second model accommodates state dependence, but not unobserved heterogeneity. The third model accommodates unobserved heterogeneity, state dependence, and heterogeneity in the state dependence effect. For all the panel-data models, we experimented with different errorcomponents structures, but none of the alternative error structures tested provided a significantly better log-likelihood value than the simple error-components structure obtained in the crosssectional analysis. In the "panel" analysis, we used 150 Halton draws per individual for individual-specific heterogeneity coefficients and 25 Halton draws per choice occasion for the inter-alternative error components (limited experimentation with higher number of draws indicated little change in coefficient estimates and log-likelihood function values).

The first model in Table 4 that incorporates unobserved heterogeneity shows that the estimated standard deviations characterizing the unobserved heterogeneity distributions for the constants are highly significant. Thus, there appears to be considerable individual-level heterogeneity in intrinsic preferences for the different alternatives. The socio-demographic variables continue to retain their significant impact. The standard deviations of the parameters on the level-of-service variables are also highly significant, indicating the presence of response heterogeneity in addition to preference heterogeneity. The estimated mean coefficient on the cost variable, along with the estimated standard deviation of the cost sensitivity across individuals, implies a negative effect of travel cost for almost all individuals. The normal 
distribution assumption for heterogeneity will necessarily imply a positive coefficient for some individuals; in the current analysis, this is estimated to be the case for less than $0.8 \%$ of individuals. Similarly, the time effect is negative for about $90 \%$ of individuals, while it is estimated to be positive for about $10 \%$ of individuals. The level-of-service coefficients in the unobserved heterogeneity model of Table 4 and the cross-sectional error-components model of Table 2 cannot be directly compared because of differences in the variances of the stochastic utility components between the two models. However, a comparison of the relative importance of the cost and time coefficients between the two models indicates clearly that time sensitivity is overstated in the cross-sectional model. Another important difference between the unobserved heterogeneity model and the cross-sectional models is the estimated scale difference in RP and SP responses. The scale factor is close to, and not significantly different from, 1 in the crosssectional models, while it is much higher than 1 and statistically different from 1 in the unobserved heterogeneity model. Thus, after accommodating unobserved heterogeneity effects, the error variance in the SP choice context is much lower than in the RP choice context. This result is significant. Most previous studies have estimated a larger error variance in the SP context than in the RP context, and have attributed this to the limited set of attributes in SP experiments or to experimental design effects. Our results suggest that the larger SP variance in earlier studies may have simply been an artifact of ignoring error correlations across repeated SP choices of the same individual. Finally, the error components generating inter-alternative error correlations across the drive alone alternatives and across the carpool alternatives weaken once unobserved heterogeneity is included (see last but one row in Table 4). This is not surprising, since unobserved heterogeneity indirectly also generates inter-alternative correlation patterns. 
The second model in Table 4 accommodates state-dependence, but ignores unobserved heterogeneity. As with the unobserved heterogeneity model, the state dependence model also shows a reduction in time sensitivity relative to cost sensitivity compared to the cross-sectional error-components model. However, the reduction in time-sensitivity is not as large as in the model with unobserved heterogeneity. Also, the scale parameter between the RP and SP choices is close to, and not significantly different from, 1 in this model. Thus, unobserved heterogeneity and state dependence have different implications regarding the sensitivity to level-of-service variables as well as regarding context differences in RP and SP choice situations. Finally, the state dependence model suggests the presence of a very significant positive impact of the current RP choice of the individual on her/his SP choices (see last row of Table 4).

The third model in Table 4 disentangles the effects of unobserved heterogeneity and state dependence. This general model includes unobserved parameter heterogeneity, state dependence, and unobserved heterogeneity in state dependence. A comparison of the third model with the first model shows a tempering of the unobserved parameter heterogeneity effects (except for the preference heterogeneity for the BART alternative, which increases slightly in the third model; see the magnitudes of the standard deviations of the parameters in Table 4). This tempering of the unobserved heterogeneity effects is intuitive, since the state dependence effect is incorrectly being manifested as unobserved heterogeneity in the first model. A consequence of the reduced heterogeneity effects in the context of the level-of-service variables is that it is even more unlikely for the cost and time parameters to be positive in the third model compared to the first model. In particular, the cost parameter is estimated to be positive for less than $0.2 \%$ of individuals and the time parameter is estimated to be positive for only about $5 \%$ of individuals. Similar to the dampening of the unobserved heterogeneity effect, there is also a 
substantial reduction in the state dependence effect in the third model relative to the second model. Overall, there is a decrease in the estimated strengths of the unobserved parameter heterogeneity and state dependence effects when they are introduced simultaneously.

The estimated parameter representing unobserved heterogeneity in state dependence is statistically significant in the third model (see last row of Table 4); the magnitude of this parameter is also large relative to the estimated mean state dependence effect. Thus, the results show that there is substantial variation in the magnitude of the state dependence effect. In fact, while the mean state dependence effect is positive, the effect is negative for $42 \%$ of individuals. It appears that both a positive effect (due to factors such as habit persistence, inertia to explore another alternative, or learning combined with risk aversion) or a negative effect (due to, for instance, variety seeking or the latent frustration of the inconvenience associated with a current alternative) may be associated with the influence of current choice on future choices.

Clearly, the results above indicate important differences among the panel data models, and between the panel data models and the cross-sectional models in Table 2. To examine the trade-offs between time and cost estimated by the panel data models, and to evaluate data fit, we present the implied time costs and fit statistics of the panel model in Table 5. The implied value of time for the models incorporating unobserved heterogeneity are computed at the mean estimated values of the time and cost parameters ${ }^{2}$. The results in Table 5 show that the estimated

${ }^{2}$ We also simulated data for two normally distributed variables with the mean and variance of the variables corresponding to the time and cost coefficients, and subsequently computed the median values of time across the simulated observations. These values are quite close to the ratio of the mean coefficients on the time and cost coefficients because the standard deviation of the cost coefficient is rather small. 
money values of time from the panel models are much smaller than the values obtained from the cross-sectional models, supporting an earlier finding by Daniels and Hensher (2000) that ignoring unobserved heterogeneity and/or state dependence can lead to inflated money values of travel time. Among the three panel-data models, the mean money value of time is overstated when only state-dependence is included. Rather coincidentally, the mean estimated money value of time is identical up to the second decimal place in the first and third models. However, the standard deviations of the cost and time coefficients are quite different across the two models (see Table 4). As we illustrate in the next section, the differences across the various models in response sensitivities, state dependence effects, and competition patterns generated by unobserved heterogeneity and error components have substantial implications for transportation policy analysis.

The fit measures in Table 5 indicate a dramatic improvement due to the inclusion of unobserved heterogeneity; the adjusted rho-bar squared value increases from 0.066 in the crosssectional error-components model to 0.527 in the model with only unobserved heterogeneity. There is also an improvement due to the inclusion of state dependence (relative to the crosssectional error components model), though the improvement is considerably lesser than when unobserved heterogeneity is included. Finally, the most general model that accommodates both unobserved heterogeneity and state dependence clearly provides the best overall fit statistic (the likelihood ratio test corresponding to a comparison of the pure unobserved heterogeneity model and the general model is 18.48 , which is larger than the chi-squared statistic with two degrees of freedom at any reasonable level of significance). 


\section{CONGESTION-PRICING POLICY SIMULATIONS}

Most transportation congestion management actions attempt to effect a change in mode choice during the peak periods or effect a change in departure time from peak to off-peak periods by influencing the level-of-service variables. For example, congestion-pricing and parking-pricing schemes rely on the use of monetary disincentives for traveling during the peak periods by the drive alone mode. In this section, we present the substantive policy implications obtained from the alternative model structures due to congestion-pricing policies targeted at Bay Area Bridge users. Specifically, we examine the impact of a $\$ 2.00$ and a $\$ 4.00$ increase in drive alone-peak (DAP) mode travel costs (the current mean DAP cost is $\$ 4.58$, so the increases above correspond to about a $44 \%$ and a $87 \%$ increase, respectively, at the mean current DAP travel cost). The impact of these congestion-pricing schemes is evaluated by modifying the cost variable for the DAP travel alternative in the RP sample, computing revised disaggregate probabilities, calculating revised expected aggregate number of individuals using each travel alternative within the RP sample, and then obtaining a percentage change from the baseline estimates. It is important to recognize that our policy simulations cannot reflect long-term changes in activitytravel behavior such as household relocation, and work activity and location changes.

In all the simulations, we use the RP alternative-specific constants and not the SP alternative-specific constants. One minor issue is that we do not have an RP constant for the carpool mode during off-peak hours (i.e., the CPO mode). This is because none of the Bay Area users in the RP sample chose the CPO mode. However, we do have the SP constant on the CPO mode. For prediction purposes, we impute a constant for the CPO mode by applying the relative difference between the CPP and CPO modes estimated from the SP data component of the joint RP-SP analysis to the RP CPP constant. In general, the resulting constant for the CPO mode is 
highly negative. Because of this, and for interpretational ease of the policy simulations as well as computation of percentage differences from the baseline, we have combined the CPP and CPO mode into a single carpool (CP) mode.

Table 6 provides the estimated percentage change (at the aggregate level) from the alternative joint RP-SP model structures and for the two levels of congestion-pricing. The five joint RP-SP models include the cross-sectional MNL, the cross-sectional error components, the panel data model with unobserved heterogeneity, the panel data model with state dependence, and the most general panel data model. As expected, all the models predict a decrease in the DAP mode and an increase in other modes due to the congestion pricing schemes. However, there are differences in the magnitude of the changes. Among the models, the MNL model overestimates the decrease in DAP share relative to the general model, while the next three models underestimate the decrease compared to the general model. The underestimation is most obvious for the state-dependence model; this may be attributed to the high inertia effect estimated in the model and the large fraction of individuals using the drive alone mode in the RP sample. In contrast, the general model estimates a much lower mean state-dependence effect and also indicates substantial "variety seeking" in the effect. Consequently, there is considerable dampening of the state dependence effect and, therefore, a higher shift away from the DAP mode. Overall, the results suggest that the MNL model will provide an overly-optimistic projection of the alleviation in traffic congestion during the peak periods due to congestionpricing schemes, while the other restrictive models in Table 6 will under-predict the alleviation in peak-period traffic congestion (compared to the general model).

The differences among the models are even more noticeable in their predictions of the increases in the market share of the non-DAP alternatives. The MNL model indicates a rather 
even increase in all other alternative shares; this is, of course, an aggregate manifestation of the individual-level IIA property. The cross-sectional error components model shows a large increase in the share of the drive alone off-peak alternative (DAO) because of the common error component corresponding to the drive alone alternatives. As indicated in Section 5, the error components structure also generates a heteroscedasticity pattern so that the transit alternatives attract more of the displacement from the DAP mode than the carpool alternatives. This is reflected in the higher percentage increases for the transit alternatives and the lower percentage increase for the carpool alternative in the error components model relative to the MNL model. The unobserved heterogeneity model shows the lowest increase in the DAO alternative of all the models. The increase in the BART alternative in this model is also smaller than the corresponding value from the MNL and error-components models. The state dependence model, on the other hand, shows the largest increase in the DAO alternative. This is to be expected because the state dependence model estimates a substantial inertia effect corresponding to the drive alone travel mode. The transit modes gain the least share in the state dependence model compared to other models. Finally, the general model shows an increase in the DAO alternative that is between those of the unobserved heterogeneity and state dependence models. The general model also predicts higher percentage increases for the carpool, ACT, and BART modes compared to the other two panel models because of the higher overall draw away from the drive alone peak alternative.

\section{SUMMARY AND CONCLUSIONS}

This paper has formulated a mixed logit model for joint RP-SP analysis that accommodates the following behavioral considerations: (a) a flexible competition pattern across alternatives, (b) 
scale difference in the RP and SP choice contexts, (c) heterogeneity across individuals in the intrinsic preferences for alternatives, (d) heterogeneity across individuals in the responsiveness to level-of-service factors, (e) state dependence of the SP choices on the RP choices, and (f) heterogeneity across individuals in the state dependence effect. To the authors' knowledge, this is one of the first research attempts to develop and apply a unified methodological framework that recognizes and accommodates all of these important behavioral considerations.

The mixed logit formulation is estimated using a maximum likelihood method. The likelihood function in the current formulation is quite different from those in previous applications of the mixed logit model. In particular, there are two levels of integration rather than one. This arises because the error components formulation that generates inter-alternative correlation operates at the choice level, while the random coefficients formulation that accommodates taste variation across individuals operates at the individual level. The maximization of the likelihood function is achieved using simulation techniques that employ quasi-random Halton draws. The Halton method, proposed for use in the estimation of discrete choice models by Bhat (1999), uses "cleverly" crafted non-random and uniformly distributed sequences in the domain of integration.

The mixed logit formulation is applied to examine the travel behavior responses of San Francisco Bay Bridge users to changes in travel conditions, including changes in bridge tolls, parking costs, travel times, transit fares, and transit service headway. Several results from the empirical analysis in the paper are noteworthy. First, the results emphasize the advantage of combining RP and SP data in travel modeling. Using only RP data results in a statistically insignificant cost coefficient, reflecting the limited variation in cost within the RP sample as well as multi-collinearity between time and cost. On the other hand, using only SP data would, in 
general, result in estimates of alternative-specific constants that are not reflective of the market shares of the alternatives; also using only SP data would not recognize state dependence effects. Joint RP-SP methods are better able to represent trade-offs in level-of-service attributes and also provide efficiency benefits in estimation by recognizing the presence of a common latent preference structure underlying the RP and SP responses. Second, the results indicate substantial heterogeneity across individuals in overall preferences for alternatives. There is also significant heterogeneity in response to level-of-service measures. Between the time and cost sensitivities, there appears to be substantially more heterogeneity across individuals in time sensitivity than in cost sensitivity. Third, the heterogeneity effects and state-dependence effects are tempered when both of these are included simultaneously. This indicates that there is a confounding of true and spurious state dependence effects when only heterogeneity or only state dependence is considered. As has already been explained earlier, disentangling these two effects is critical for transportation policy analysis. Fourth, ignoring unobserved heterogeneity and/or state dependence effects leads to an overestimation of time sensitivity; thus, using "cross-sectional" methods of analysis that ignore the repeated-choice nature of SP responses and the dependence of SP responses on RP responses lead to biased estimates of the effects of level-of-service variables in the current empirical context. Fifth, there is a dramatic improvement in data fit when one introduces unobserved heterogeneity. The rho-bar squared value increases from about 0.07 to about 0.53 when unobserved heterogeneity is introduced. There is also a considerable improvement in data fit when state-dependence is introduced, but the improvement is small relative to the case when unobserved heterogeneity is introduced. Further, the fit improvement when state dependence is introduced in a model with unobserved heterogeneity is relatively marginal. This result, along with the small changes in parameter estimates and implied money 
values of time between a model with only unobserved heterogeneity and a model with both unobserved heterogeneity and state dependence, suggests that unobserved heterogeneity effects are much stronger in the current empirical context than state-dependence effects. This is not to suggest that state-dependence effects may be ignored; it is intended to characterize the relative strengths of the two effects. It is possible that the unobserved heterogeneity effects will not be so dominating in a different empirical context or if more detailed demographic/attitudinal data becomes available for inclusion in the model specifications. Sixth, the results indicate substantial heterogeneity across individuals in the state-dependence effect; it appears that both a positive effect (due to factors such as habit persistence, inertia to explore another alternative, or learning combined with risk aversion) or a negative effect (due to, for instance, variety seeking or the latent frustration of the inconvenience associated with a current alternative) may be associated with the influence of current choice on future choices. Seventh, there is a dramatic increase in the estimated scale difference in RP and SP responses when unobserved heterogeneity is accommodated; that is, after accommodating unobserved heterogeneity effects, the error variance in the SP choice context is much lower than in the RP choice context. This result is quite different from those in most earlier RP-SP studies, which have estimated a larger error variance in the SP context than in the RP context or have found the error variances to not be significantly different. These earlier studies have attributed a higher SP error variance to the limited set of attributes in SP experiments or to experimental design effects. Our results suggest that the larger SP variance in earlier studies may have been an artifact of ignoring error correlations across repeated SP choices from the same individual. Thus, it is possible that the SP choice context provides a very focused setting compared to an RP context, with relatively little room for measurement error or imputation of variable values. This, in combination with recent 
studies that suggest the ability of consumers to systematically evaluate even rather complex hypothetical scenarios (see Louviere and Hensher, 2000), points toward using SP experiments as the main data source for analysis and supplementing with small samples of RP data for anchoring with actual market activity.

The substantive congestion-pricing policy implications on the shares of the various travel alternatives are quite different among the alternative models. The results suggest that the crosssectional MNL model will provide an overly-optimistic projection of the alleviation in traffic congestion during the peak periods due to congestion-pricing schemes, while the other restrictive models (the cross-sectional error-components model, the panel-data model with unobserved heterogeneity only, and the panel data model with state dependence only) will under-predict the alleviation in peak-period traffic congestion (compared to the general model). The differences between the general model and the restrictive structures are particularly noticeable in their predictions of the increases in the market share of the non-DAP alternatives. These results highlight the need to include (or at least test for) flexible inter-alternative error structures, unobserved heterogeneity, state dependence, and heterogeneity in the state dependence effects within the context of a unified methodological framework to assist informed policy decisionmaking.

\section{ACKNOWLEDGEMENTS}

The authors appreciate the useful comments of David Hensher and two anonymous referees on an earlier version of the paper. Ken Vaughn provided the San Francisco Bay area data and clarified data issues. Lisa Weyant helped with typesetting and formatting the paper. 


\section{REFERENCES}

Ailawadi, K.L., Gedenk, K., and S.A. Neslin. Heterogeneity and Purchase Event Feedback in Choice Models: An Empirical Analysis with Implications for Model Buildings. International Journal of Research in Marketing, Vol. 16, 1999, pp.177-198.

Ben-Akiva, M. and S. Lerman. Discrete Choice Analysis: Theory and Application to Travel Demand. MIT Press, Cambridge, Massachusetts, 1985.

Ben-Akiva, M. and T. Morikawa. Estimation of Travel Demand Models from Multiple Data Sources. Proceedings from the $11^{\text {th }}$ International Symposium on Transportation and Traffic Theory, Yokohama, Japan, Elsevier, New York, 1990, pp. 461-476.

Ben-Akiva, M. and D. Bolduc. Multinomial Probit with a Logit Kernel and a General

Parametric Specification of the Covariance Structure. Working paper, Department of Civil and Environmental Engineering, Massachusetts Institute of Technology, Cambridge, Massachusetts and Departement d' economique, Universite Laval, SainteFoy, Quebec, Canada, 1996.

Ben-Akiva, M., Morikawa, T., and F. Shiroishi. Analysis of the Reliability of Preference Ranking Data. Journal of Business Research, Vol. 24, 1992, pp. 149-164.

Bhat, C.R. A Heteroscedastic Extreme Value Model of Intercity Travel Mode Choice. Transportation Research, Vol. 29B, No. 6, 1995, pp. 471-483.

Bhat, C.R. Accommodating Variations in Responsiveness to Level-of-Service Measures in Travel Mode Choice Modeling. Transportation Research, Vol. 32A, 1998, pp. 495-507.

Bhat, C.R. Quasi-Random Maximum Simulated Likelihood Estimation of the Mixed Multinomial Logit Model. Forthcoming, Transportation Research, 2001. 
Bhat, C.R. Flexible Model Structures for Discrete Choice Analysis. In D.A. Hensher, K.J. Button (eds.) Handbook of Transport Modelling, Pergamon Press, Oxford, 2000, pp. 7190.

Brownstone, D., and K. Train. Forecasting New Product Penetration with Flexible Substitution Patterns. Journal of Econometrics, Vol. 89, 1999, pp. 109-129.

Brownstone, D., Bunch, D.S., and K. Train. Joint Mixed Logit Models of Stated and Revealed Preferences for Alternative-Fuel Vehicles. Transportation Research, Vol. 34B, 2000, pp. $315-338$.

Brownstone, D., Bunch, D.S., Golob, T., Ren. A Transactions Choice Model for Forecasting Demand for Alternative-Fuel Vehicles. In S. McMullen (ed.) Research in Transportation Economics, 4, JAI Press Inc., 1996, pp. 87-129.

Chamberlain, G. Analysis of Covariance With Qualitative Data. Review of Economic Studies, Vol. 47, 1980, pp. 225-238.

Daniels, R. and D.A. Hensher. Valuation of Environmental Impacts of Transportation Projects: The Challenge of Self-Interest Proximity. Forthcoming, Journal of Transport Economics and Policy.

Degeratu, A.M. Estimation Bias in Choice Models with Last Choice Feedback. International Journal of Research in Marketing, Vol.16, 1999, pp. 285-306.

Diggle, P.J., Liang, K.-Y., and S.L. Zeger. Analysis of Longitudinal Data. Clarendon Press, Oxford, U.K., 1994.

Gunn, H.F., Bradley, M.A., and D.A. Hensher. High Speed Rail Market Projection: Survey Design and Analysis, Transportation, Vol. 19, 1992, pp. 117-139. 
Hajivassiliou, A., and P.A. Ruud. Classical Estimation Methods for LDV Models Using Simulation. In R.F. Engle, D.L. McFadden (eds.) Handbook of Econometrics, 4, North Holland, Amsterdam, 1994, pp. 2383-2441.

Heckman, J.J. The Incidental Parameters Problem and the Problem of Initial Conditions in Estimating a Discrete Time-Discrete Data Stochastic Process. In C.F. Manski, D. McFadden (eds.) Structural Analysis of Discrete Data with Econometric Applications, MIT Press, Cambridge, Massachusetts, 1981, pp. 179-195.

Hensher, D.A. The Valuation of Travel Time Savings for Urban Car Drivers: Evaluating Alternative Model Specifications. Technical Paper, Institute of Transport Studies, The University of Sydney, Australia, 1999.

Hensher, D.A. Measurement of the Valuation of Travel Time Savings. Special issue in memory of Michael E. Beesley, forthcoming, Transportation Economics and Policy, 2000.

Hensher, D.A., and W. Greene. Choosing Between Conventional, Electric, and UPG/LNG Vehicles in Single Vehicle Households. Technical paper, Institute of Transport Studies, University of Sydney, Australia, 2000.

Hensher, D.A., Louviere, J., and J. Swait. Combining Sources of Preference Data. Journal of Econometrics, Vol. 89, 1999, pp. 197-221.

Hsiao, C. Analysis of Panel Data. Cambridge University Press, Cambridge, Massachusetts, 1986.

Keane, M.P. Modeling Heterogeneity and Stated Dependence in Consumer Choice Behavior. American Statistical Association Journal of Business \& Economic Statistics, Vol. 15, No. 3, 1997, pp. 310-327. 
Koppelman, F.S, and V. Sethi. Closed-Form Discrete-Choice Models. In D.A. Hensher, K.J. Button (eds.) Handbook of Transport Modelling, Pergamon Press, Oxford, 2000, pp. 211227.

Koppelman, F.S., Bhat, C.R., and J.L. Schofer. Market Research Evaluation of Actions to Reduce Traffic Congestion: Commuter Travel Behavior and Response to Demand Reduction Actions. Transportation Research, Vol. 27A, No. 5, 1993, pp. 383-393.

Lee, L.-F. On the Efficiency of Methods of Simulated Moments and Maximum Simulated Likelihood Estimation of Discrete Response Models. Econometric Theory, Vol. 8, 1992, pp. 518-552.

Louviere, J.J., and D.A. Hensher. Combining Preference Data, (Resource Paper, $9^{\text {th }}$ International Association for Travel Behaviour Research Conference, Gold Coast, Queensland, Australia, July 2000). Forthcoming in The Leading Edge of Travel Behaviour Research, D.A. Hensher, J. King (eds), Pergamon Press, Oxford.

Louviere, J.J., Meyer, R.J., Bunch, D.S., Carson, R., Dellaert, B., Hanemann, W.M., Hensher, D.A., and J. Irwin. Combining Sources of Preference Data for Modeling Complex Decision Processes. Marketing Letters, Vol. 10, No. 3, 1999, pp. 205-217.

Luce, R.D., and P. Suppes. Preference, Utility, and Subjective Probability. In R.D. Luce, R.R. Bush, E. Galanter (eds.) Handbook of Mathematical Psychology, John Wiley \& Sons, New York, 3, 1965, pp. 249-410.

McFadden, D. Modeling the Choice of Residential Location. In A. Karlquist (ed.) Spatial Interaction Theory and Residential Location, North Holland, Amsterdam, 1978, pp. 7596. 
McFadden, D., and K. Train. Mixed MNL Models for Discrete Response. Department of Economics, University of California at Berkeley, 1998.

Morikawa, T. Correcting State Dependence and Serial Correlation in the RP/SP Combined Estimation Method. Transportation, Vol. 21, 1994, pp. 153-165.

NuStats Research and Consulting. 1996 Bay Area Travel Study: Final Report submitted to the Metropolitan Transportation Commission, 1996.

Swait, J., and J.J. Louviere. The Role of the Scale Parameter in the Estimation and Comparison of Multinomial Logit Models. Journal of Marketing Research, Vol. 30, 1993, pp. 305314.

Train, K. Halton Sequences for Mixed Logit. Technical Paper, Department of Economics, University of California, Berkeley, 1999. 


\section{LIST OF TABLES}

TABLE 1 Availability and Choice Shares of Alternatives

TABLE 2 Cross-sectional Model Estimation Results

TABLE 3 Cross-sectional Models: Money Values of Time and Fit-Statistics

TABLE 4 Panel Data Model Estimation Results

TABLE 5 Panel-Data Models: Money Values of Time and Fit-Statistics

TABLE 6 Congestion Pricing Policy Simulations 
TABLE 1 Availability and Choice Shares of Alternatives

\begin{tabular}{|c|c|c|c|c|c|c|}
\hline \multirow{2}{*}{ Alternative } & \multicolumn{2}{|c|}{ Revealed Preference Sample } & \multicolumn{2}{|c|}{ Stated Preference Sample } & \multicolumn{2}{|c|}{ Joint Sample } \\
\hline & $\begin{array}{c}\text { Availability } \\
\text { Shares }^{1}\end{array}$ & Choice Shares & $\begin{array}{c}\text { Availability } \\
\text { Shares }{ }^{1}\end{array}$ & Choice Shares & $\begin{array}{l}\text { Availability } \\
\text { Shares }^{1}\end{array}$ & Choice Shares \\
\hline Drive alone - peak (DAP) & 1.000 & 0.617 & 1.000 & 0.226 & 1.000 & 0.270 \\
\hline Drive alone - offpeak (DAO) & 1.000 & 0.081 & 1.000 & 0.298 & 1.000 & 0.273 \\
\hline Carpool - peak (CPP) & 1.000 & 0.037 & 1.000 & 0.169 & 1.000 & 0.154 \\
\hline Carpool - offpeak (CPO) & 1.000 & 0.000 & 1.000 & 0.014 & 1.000 & 0.012 \\
\hline Alameda County transit (ACT) & 0.463 & 0.037 & 0.543 & 0.065 & 0.534 & 0.061 \\
\hline Bay area rapid transit (BART) & 0.904 & 0.228 & 0.912 & 0.228 & 0.911 & 0.228 \\
\hline Sample Size & \multicolumn{2}{|c|}{136} & \multicolumn{2}{|c|}{1068} & \multicolumn{2}{|c|}{1204} \\
\hline
\end{tabular}

${ }^{1}$ The availability shares represent the share of observations in the sample for which the alternative is available. 
TABLE 2 Cross-sectional Model Estimation Results

\begin{tabular}{|c|c|c|c|c|c|c|c|c|}
\hline \multirow[t]{2}{*}{ Variable } & \multicolumn{2}{|c|}{ RP-Sample MNL } & \multicolumn{2}{|c|}{ SP-Sample MNL } & \multicolumn{2}{|c|}{ Joint RP-SP MNL } & \multicolumn{2}{|c|}{$\begin{array}{l}\text { Joint RP-SP Error } \\
\text { Components Model }\end{array}$} \\
\hline & Parm. & t-stat. & Parm. & t-stat. & Parm. & t-stat. & Parm. & t-stat. \\
\hline \multicolumn{9}{|l|}{ Constants } \\
\hline \multicolumn{9}{|l|}{ RP Sample } \\
\hline Drive alone off-peak & -0.277 & -0.32 & - & - & 0.602 & 0.93 & 0.539 & 0.60 \\
\hline Carpool peak & -1.165 & -1.20 & - & - & -0.554 & -0.81 & -2.040 & -2.11 \\
\hline $\mathrm{ACT}$ & 0.712 & 0.49 & - & - & -0.144 & -0.20 & -0.303 & -0.37 \\
\hline BART & 2.328 & 2.72 & - & - & 2.417 & 3.34 & 2.182 & 2.19 \\
\hline \multicolumn{9}{|l|}{ SP Sample } \\
\hline Drive alone off-peak & - & - & 3.029 & 6.84 & 2.536 & 6.91 & 2.476 & 6.41 \\
\hline Carpool peak & - & - & 1.935 & 4.05 & 2.390 & 4.73 & 3.320 & 3.80 \\
\hline Carpool off-peak & - & - & -0.423 & -0.79 & -0.994 & -1.37 & -0.816 & -1.21 \\
\hline $\mathrm{ACT}$ & - & - & 1.310 & 2.25 & 1.367 & 2.53 & 1.850 & 2.96 \\
\hline BART & - & - & 3.196 & 6.79 & 0.987 & 3.49 & 1.216 & 2.67 \\
\hline \multicolumn{9}{|l|}{ Socio Demographic Variables } \\
\hline \multicolumn{9}{|l|}{ Vehicles per worker in household } \\
\hline Drive alone alternatives & 1.057 & 3.03 & 0.444 & 5.22 & 0.573 & 3.39 & 0.748 & 2.35 \\
\hline \multicolumn{9}{|l|}{ Male } \\
\hline Drive alone peak & 0.681 & 1.67 & 0.647 & 3.73 & 0.771 & 3.23 & 0.666 & 2.43 \\
\hline \multicolumn{9}{|l|}{ Employed } \\
\hline Drive alone peak & 0.900 & 1.60 & 1.789 & 4.48 & 1.638 & 4.42 & 1.730 & 2.86 \\
\hline \multicolumn{9}{|l|}{ Income (in U.S. dollars/year) x 10-5 } \\
\hline Drive alone peak & 0.454 & 0.71 & 0.660 & 2.69 & 0.761 & 2.47 & 0.711 & 2.10 \\
\hline ACT & 1.596 & 1.06 & 2.176 & 5.18 & 2.526 & 3.48 & 2.362 & 2.67 \\
\hline \multicolumn{9}{|l|}{ Level of Service Variables } \\
\hline Travel Time (in mins.) x $10-2$ & -5.161 & -3.55 & -2.166 & -7.37 & -2.792 & -3.69 & -2.668 & -2.58 \\
\hline Cost & -0.155 & -0.76 & -0.121 & -4.54 & -0.147 & -3.08 & -0.157 & -2.38 \\
\hline SP-to-RP scale factor ${ }^{1}$ & - & - & - & - & 0.831 & 0.21 & 0.779 & 0.26 \\
\hline \multicolumn{9}{|l|}{ Error Components } \\
\hline Drive alone alternatives (DAP and DAO) & - & - & - & - & - & - & 1.850 & 2.18 \\
\hline Carpool alternatives (CPP and CPO) & - & - & - & - & - & - & 1.850 & 2.18 \\
\hline
\end{tabular}

${ }^{1}$ The t-statistic corresponding to the scale factor is computed with respect to a value of 1 ; a value of 1 indicates no scale difference in the RP and SP choice contexts. 
TABLE 3 Cross-sectional Models: Money Values of Time and Fit-Statistics

\begin{tabular}{|c|c|c|c|c|}
\hline Parameter & RP-Sample MNL & SP-Sample MNL & Joint RP-SP MNL & $\begin{array}{l}\text { Joint RP-SP Error } \\
\text { Component Model }\end{array}$ \\
\hline Implied money value of time $(\$ / \mathrm{hr})$ & 19.95 & 10.74 & 11.38 & 10.17 \\
\hline Log-likelihood at zero & -199.05 & -1789.43 & -1988.48 & -1988.48 \\
\hline Log-likelihood with constants only & -139.41 & -1596.21 & -1735.62 & -1735.62 \\
\hline Log-likelihood at convergence & -120.43 & -1493.81 & -1618.65 & -1612.66 \\
\hline Number of parameters excluding constants & 7 & 7 & 8 & 9 \\
\hline Adjusted Rho-bar squared ${ }^{1}$ & 0.086 & 0.06 & 0.063 & 0.066 \\
\hline
\end{tabular}

${ }^{1}$ The adjusted rho-bar squared value $\left(\bar{\rho}^{2}\right)$ is computed as follows: $\bar{\rho}^{2}=1-[(L(\hat{\beta})-K) / L(C)]$ where $L(\hat{\beta})$ is the log-likelihood value at convergence, $L(C)$ is the log-likelihood value with only the constants, and $K$ is the number of estimated parameters excluding the constants. 
TABLE 4 Panel Data Model Estimation Results

\begin{tabular}{|c|c|c|c|c|c|c|}
\hline \multirow[t]{2}{*}{ Variable } & \multicolumn{2}{|c|}{$\begin{array}{c}\text { Unobserved } \\
\text { Heterogeneity Model }\end{array}$} & \multicolumn{2}{|c|}{$\begin{array}{l}\text { State Dependence } \\
\text { Model }\end{array}$} & \multicolumn{2}{|c|}{$\begin{array}{c}\text { Unobs. Heterogeneity } \\
+ \text { State Dependence } \\
\text { Model }\end{array}$} \\
\hline & Parm. & t-stat. & Parm. & t-stat. & Parm. & t-stat. \\
\hline Constants & & & & & & \\
\hline $\begin{array}{l}\text { Mean effects - RP Sample } \\
\text { Drive alone off-peak } \\
\text { Carpool peak } \\
\text { ACT } \\
\text { BART }\end{array}$ & $\begin{array}{r}-0.773 \\
-3.030 \\
-2.423 \\
0.665\end{array}$ & $\begin{array}{r}-1.40 \\
-3.77 \\
-2.41 \\
1.12\end{array}$ & $\begin{array}{r}-0.604 \\
-1.813 \\
-0.774 \\
1.818\end{array}$ & $\begin{array}{r}0.68 \\
-2.24 \\
-1.02 \\
2.11\end{array}$ & $\begin{array}{r}-0.332 \\
-2.031 \\
-1.673 \\
0.619\end{array}$ & $\begin{array}{r}-0.52 \\
-2.89 \\
-1.87 \\
0.95\end{array}$ \\
\hline $\begin{array}{l}\text { Mean effects - SP Sample } \\
\text { Drive alone off-peak } \\
\text { Carpool peak } \\
\text { Carpool off-peak } \\
\text { ACT } \\
\text { BART }\end{array}$ & $\begin{array}{r}2.478 \\
2.610 \\
-3.469 \\
1.237 \\
0.601\end{array}$ & $\begin{array}{r}6.59 \\
4.30 \\
-2.29 \\
1.80 \\
1.74\end{array}$ & $\begin{array}{r}2.481 \\
4.150 \\
-0.123 \\
2.567 \\
1.622\end{array}$ & $\begin{array}{r}6.41 \\
4.25 \\
-0.21 \\
3.72 \\
3.19\end{array}$ & $\begin{array}{r}2.499 \\
2.787 \\
-1.157 \\
1.374 \\
0.725\end{array}$ & $\begin{array}{r}6.81 \\
4.90 \\
-1.40 \\
2.15 \\
2.16\end{array}$ \\
\hline $\begin{array}{l}\text { Standard deviations } \\
\text { Drive alone off-peak } \\
\text { Carpool peak } \\
\text { Carpool off-peak } \\
\text { ACT } \\
\text { BART }\end{array}$ & $\begin{array}{l}1.301 \\
1.596 \\
2.512 \\
1.408 \\
1.493\end{array}$ & $\begin{array}{l}4.39 \\
4.03 \\
2.83 \\
3.32 \\
4.98\end{array}$ & $\begin{array}{l}- \\
- \\
- \\
- \\
-\end{array}$ & $\begin{array}{l}- \\
- \\
- \\
- \\
-\end{array}$ & $\begin{array}{l}1.003 \\
0.895 \\
1.374 \\
1.158 \\
1.512\end{array}$ & $\begin{array}{l}4.06 \\
3.72 \\
3.13 \\
3.04 \\
4.04\end{array}$ \\
\hline Socio-demographic variables & & & & & & \\
\hline $\begin{array}{l}\text { Vehicles per worker in household } \\
\text { Drive alone alternatives } \\
\text { Male }\end{array}$ & 0.233 & 1.72 & 0.360 & 1.94 & 0.072 & 0.77 \\
\hline & 0.337 & 2.14 & 0.654 & 2.39 & 0.592 & 3.21 \\
\hline $\begin{array}{l}\text { Drive alone peak } \\
\text { Income ( in U.S. dollars/years ) x 10-5 }\end{array}$ & 0.876 & 2.38 & 1.781 & 2.99 & 1.169 & 2.45 \\
\hline $\begin{array}{l}\text { Drive alone peak } \\
\text { ACT }\end{array}$ & $\begin{array}{l}0.617 \\
2.118 \\
\end{array}$ & $\begin{array}{l}2.62 \\
2.16 \\
\end{array}$ & $\begin{array}{l}0.752 \\
2.404 \\
\end{array}$ & $\begin{array}{l}2.08 \\
2.73 \\
\end{array}$ & $\begin{array}{l}0.557 \\
1.672 \\
\end{array}$ & $\begin{array}{l}2.38 \\
2.46 \\
\end{array}$ \\
\hline Level of service variables & & & & & & \\
\hline $\begin{array}{l}\text { Mean effects } \\
\text { Travel time (in mins.) x 10-2 } \\
\text { Travel cost (in U.S. \$) } \\
\text { Standard deviations } \\
\text { Travel time (in mins.) x 10-2 } \\
\text { Travel cost (in U.S. \$) } \\
\end{array}$ & $\begin{array}{l}-1.465 \\
-0.167\end{array}$ & $\begin{array}{l}-4.37 \\
-4.51 \\
2.63 \\
3.20 \\
\end{array}$ & $\begin{array}{c}-1.919 \\
-0.157 \\
- \\
- \\
\end{array}$ & $\begin{array}{l}-2.47 \\
-2.37\end{array}$ & $\begin{array}{r}-1.443 \\
-0.165 \\
\\
0.895 \\
0.057 \\
\end{array}$ & $\begin{array}{r}-3.48 \\
-3.54 \\
\\
2.84 \\
3.56 \\
\end{array}$ \\
\hline SP to RP scale factor ${ }^{1}$ & 4.849 & 3.68 & 0.961 & 0.12 & 5.665 & 3.24 \\
\hline $\begin{array}{l}\text { Error components } \\
\text { Drive alone alternatives (DAP and DAO) } \\
\text { Carpool alternatives (CPP and CPO) }\end{array}$ & $\begin{array}{l}0.082 \\
0.082\end{array}$ & $\begin{array}{l}1.54 \\
1.54\end{array}$ & $\begin{array}{l}1.436 \\
1.436\end{array}$ & $\begin{array}{l}2.25 \\
2.25\end{array}$ & $\begin{array}{l}0.049 \\
0.049\end{array}$ & $\begin{array}{l}1.09 \\
1.09\end{array}$ \\
\hline $\begin{array}{l}\text { State dependence effects } \\
\text { Mean } \\
\text { Standard deviation }\end{array}$ & - & - & $\begin{array}{c}1.647 \\
-\end{array}$ & $\begin{array}{c}2.69 \\
-\end{array}$ & $\begin{array}{l}0.179 \\
0.855\end{array}$ & $\begin{array}{l}2.28 \\
2.84\end{array}$ \\
\hline
\end{tabular}

${ }^{1}$ The t-statistic corresponding to the scale factor is computed with respect to a value of 1; a value of 1 indicates no scale difference in the RP and SP choice contexts. 
TABLE 5 Panel-Data Models: Money Values of Time and Fit-Statistics

\begin{tabular}{|c|c|c|c|}
\hline Parameter & $\begin{array}{l}\text { Unobserved Heterogeneity } \\
\text { Model }\end{array}$ & State Dependence Model & $\begin{array}{c}\text { Unobserved Heterogeneity } \\
\text { Model + State Dependence } \\
\text { Model }\end{array}$ \\
\hline Implied money value of time $(\$ / \mathrm{hr})$ & 5.26 & 7.33 & 5.26 \\
\hline Log-likelihood at zero & -1988.48 & -1988.48 & -1988.48 \\
\hline Log-likelihood with constants only & -1735.62 & -1735.62 & -1735.62 \\
\hline Log-likelihood at convergence & -804.06 & -1500.06 & -794.82 \\
\hline Number of parameters excluding constants & 16 & 10 & 18 \\
\hline Adjusted rho-bar squared value ${ }^{1}$ & 0.527 & 0.130 & 0.532 \\
\hline
\end{tabular}

${ }^{1}$ The adjusted rho-bar squared value $\left(\bar{\rho}^{2}\right)$ is computed as follows: $\bar{\rho}^{2}=1-[(L(\hat{\beta})-K) / L(C)]$ where $L(\hat{\beta})$ is the log-likelihood value at convergence, $L(C)$ is the log-likelihood value with only the constants, and $K$ is the number of estimated parameters excluding the constants. 
TABLE 6 Congestion Pricing Policy Simulations

\begin{tabular}{|c|c|c|c|c|c|c|}
\hline \multirow{3}{*}{ Pricing Level } & \multirow{3}{*}{ Alternative } & \multicolumn{5}{|c|}{ Percentage change estimated by... } \\
\hline & & \multicolumn{2}{|c|}{ Cross-sectional Models } & \multicolumn{3}{|c|}{ Panel Data Models } \\
\hline & & MNL & Error-components & $\begin{array}{l}\text { Unobserved } \\
\text { Heterogeneity }\end{array}$ & State Dependence & $\begin{array}{c}\text { Unobserved } \\
\text { Heterogeneity + } \\
\text { State Dependence }\end{array}$ \\
\hline \multirow{5}{*}{$\$ 2.00$} & Drive alone - peak & -9.43 & -8.77 & -8.80 & -7.01 & -9.06 \\
\hline & Drive alone - off-peak & 17.54 & 20.52 & 10.71 & 23.04 & 13.30 \\
\hline & Carpool & 15.50 & 12.54 & 16.74 & 13.11 & 19.43 \\
\hline & ACT & 15.66 & 18.32 & 16.56 & 14.94 & 18.09 \\
\hline & BART & 14.24 & 15.82 & 11.80 & 10.22 & 13.92 \\
\hline \multirow{5}{*}{$\$ 4.00$} & Drive alone - peak & -19.16 & -17.96 & -17.87 & -14.31 & -18.44 \\
\hline & Drive alone - off-peak & 36.40 & 43.34 & 21.11 & 49.83 & 26.39 \\
\hline & Carpool & 31.82 & 26.48 & 32.16 & 27.18 & 39.98 \\
\hline & ACT & 31.53 & 35.11 & 33.81 & 27.30 & 36.91 \\
\hline & BART & 28.65 & 32.52 & 24.34 & 20.29 & 28.66 \\
\hline
\end{tabular}

